\title{
Genetic Circuit for the Early Warning of Lung Cancer using iBioSim
}

\author{
Xiang WANG ${ }^{1, a}$, Xiao- Cui WANG ${ }^{1}$, Jing- Bo JIANG ${ }^{2}$, Jin- Jing SUN¹, Shu- Song PANG \\ Han- Hong $\mathrm{HE}^{1}$ and Wei- Ke WANG ${ }^{1}$ \\ ${ }^{1}$ School of Electronic and Information Engineering, Beihang University, Beijing, 100191, China \\ ${ }^{2}$ Honors College of Beihang University, Beihang University, Beijing, 100191, China \\ a Corresponding author: wxiang@buaa.edu.cn
}

\begin{abstract}
With the development of molecular biology and gene-engineering technology, gene diagnosis has been an emerging approach for modern life sciences. Biological marker, recognized as the hot topic in the molecular and gene fields, has important values in early diagnosis, malignant tumour stage, treatment and therapeutic efficacy evaluation. The design of markers detection genetic circuit system for lung cancer is presented as a new method to provide basis for early warning and therapy. The system consists of three singlemarker detection circuits and an integration circuit. The single-marker detection circuit provides an instantaneous low level when target marker's concentration reaches the threshold. The integration circuit uses gene and gate to complete the output data fusion from single-marker detection circuit through logic operations to finish the combined detection. All the structure is modelled and analyzed by iBioSim through the biochemical reactions of different gene circuits. The experimental result indicates that the whole lung cancer detection system can realize joint detection of tumor markers with good stability and sensitivity.
\end{abstract}

\section{Introduction}

Nowadays, lung cancer has become one of the most prevalent and deadly cancers worldwide, both the occurrence rate and the mortality rate remain at a high level. For instance, in China, it is estimated that there are as many as 650 thousand new lung cancer cases in 2012, occupying $22 \%$ of all cancer cases, according to recent published Cancer Fact Sheets by World Health Organization in Glob can project 2012 [1]. Especially in recent years, due to more and more serious air pollution, China's central and eastern regions faces some of the world's worst smog, which will increase the risk of lung cancer. Because symptoms of lung cancer resemble those of other diseases such as phthisis or pulmonary infection, it is possible to misdiagnose clinically and even to lose the optimal opportunity for the treatment. So far, conventional detecting methods includes chest CT examination, bronchoscope, lung puncture test and sputum cytology examination, requiring high testing costs and expensive apparatuses [2-3]. All these methods rely on doctors' judgment based on experiments, and are time and money consuming to some extent. This current situation calls for new technologies to realize more efficient and accurate lung cancer detection, and the development of genetic circuit meets this need.

Tumor Marker detection has become a hit idea in achieving early-stage prediction and treatment of cancer. It is an indicator of physiological and pathological state, produced by the cancer tissue itself or by the body in response to cancer growth. Compared to the traditional detection methods which usually have considerable experimental requirements and costs, tumor marker can not only explore the pathogenesis at the molecular level, but also have unique advantages in accurate and sensitive evaluation of early, low levels damage, which provides an early warning of cancer [4]. Many markers such as: CEA and CYFR21-1 have been found to play important roles in the diagnosis, treatment and prognosis of lung disease [5-6]. Thus, taking advantage of the genetic circuits inside cells, we can achieve more sensitive and economical tumor marker detection with good real-time performance.

The application of gene circuit has grown considerably and has chartered many notable achievements, offering new tools for the detection and prevention of lung cancer [7-8]. Researchers now have already constructed gate circuits, latch, and inverting amplifier in the gene circuit to build a complex regulation network to simulate the biochemical reactions in organisms [9-10]. Collins constructed a genetic toggle switch containing promoter that drives the expression of mutually inhibitory transcriptional repressors [11]. Anderson designed an AND logic gate based on the transcription of a gene, for which translation is dependent on the co-transcription of an engineered tRNA [12]. Tam sir and Moon finished comprehensive engineering of robust transcriptional logic including all 16 elementary logic gates, as well as the engineering of a multiple input logic network using a multitier transcriptional cascade 
[13-14]. Chau used protein signalling circuits to produce spatial polarization in yeast by engineering circuits from components that can be self-organized into localized distributions [15]. We propose a genetic circuit network to realize the detection of breast cancer [16].

In this paper, we focus on constructing and simulating a genetic circuit in detecting lung cancer tumor markers so as to realize efficient and accuracy detection. The discussion is on cell level, so we only consider the chemical and biological reactions inside the cell, assuming no other interferences occur to react with reactants in our models. Our discussion contains mainly two parts: the single marker detection circuit and the combined detection circuit. The former part, basis of the whole circuit, detects the concentration of one certain target marker, and then outputs the result to the later part as its input with further logical operation and the emergence of the warning signal. Compared with our previous design on breast cancer, we innovatively present the influence of rRNA and mRNA concentration in the single marker detection circuit and threshold selection in the whole system to improve the detection accuracy and stability.

\section{Single Marker Detection Circuit}

The inverter genetic circuit is the core structure of single marker detection circuit, and is also used in the integration circuit. Generally, biochemical reactions in organisms include four types: transcription and translation process, regulation of protein multimerization process, regulation protein binding process and the deconstruction of different proteins and mRNA. Based on the biochemical reactions, we establish the iBioSim model of the inverter, shown in figure 1. In our design, we mainly use the inverter genetic circuit to detect the changes of the three markers' concentration. Since tumor markers are often RNA or have similar function, we assume the input signal is RNA.

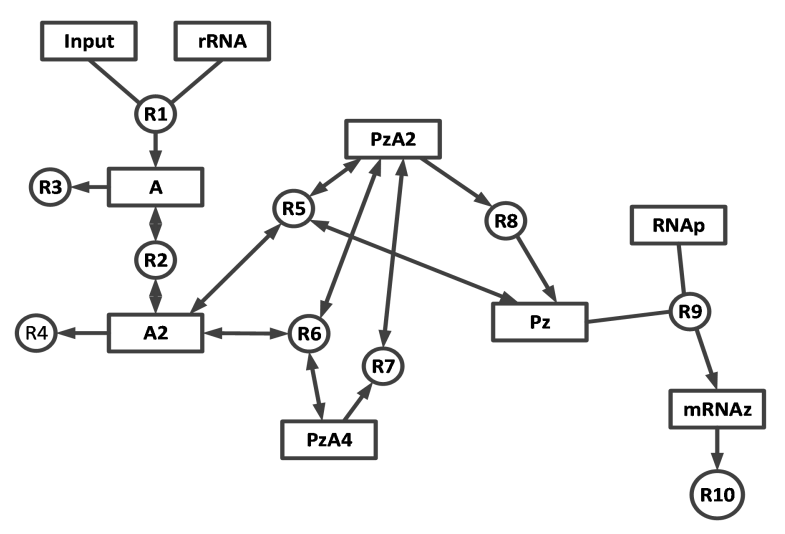

Figure 1. The SBML Model of The Genetic Inverter Constructed Using Ibiosim

In the single marker detection circuit, the input module is responsible for detecting input signal constantly and pass on the information. Specifically, we find out that the concentration of rRNA can influence the output response of single index detection module, which will change the threshold and affect the stability of the circuit. The whole system will be too sensitive and susceptible to interference if the rRNA concentration is too high; the next stage circuit cannot detect the input signal and be normally driven if the rRNA concentration is too low. And for this reason, we need to select the appropriate threshold in the final integration circuit.

\section{Integration AND Gate Circuit}

Different markers show varying sensitivity to the prediction of metastatic recurrence, so the integration circuit is put forward to build a multi-index monitor circuit network to implement the output data fusion of single-marker detection circuit through logic operations, just like the digital logic circuit. Once the integration circuit receives a signal from the single-index circuit, the genetic circuit in cells will be triggered, with green fluorescent protein (GFP) produced in large quantities to provide an early-warning of lung cancer. In our design, the integration circuit is implemented by AND logical operations constructed from a NAND gate followed by an inverter. In this case, GFP is produced only when both the two inputs of NAND gate are present. The main principle of the algorithm is shown as below. Similar to the inverter, an SBML model of the genetic and-gate is constructed using iBioSim, as shown in figure 2.

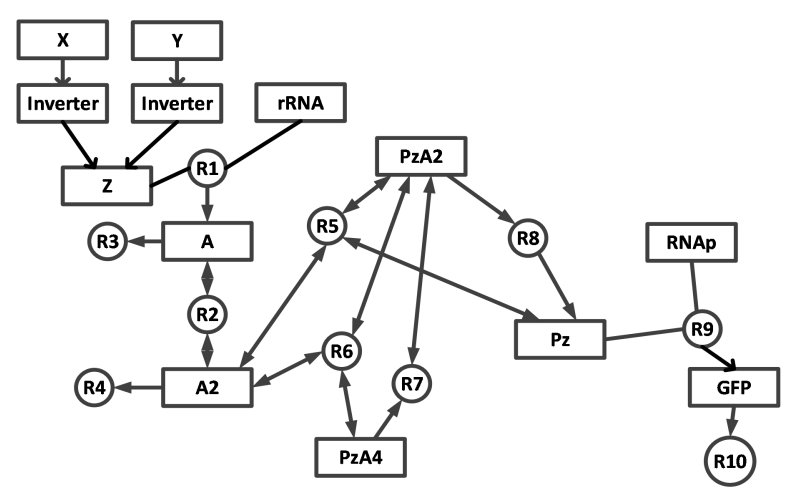

Figure 2. The SBML Model of The Genetic And-Gate Constructed Using Ibiosim

\section{The Whole Joint Detection Circuit System}

The novel complete design model for detecting lung cancer is a comprehensive system integrating of singleindex circuit and the and-gate integration circuit, and includes two parts: the concentration test (measurement) and the logical operation. The whole structure is shown in figure 3. Combined detection of biomarkers associated with lung disease and cancer is proposed to increase the reliability and accuracy of the system. According to the clinical efficacy research, taking TPS $>80 \mathrm{U} / \mathrm{L}, \mathrm{CEA}>$ $5 \mathrm{ng} / \mathrm{mL}$ and CYFR2 $1-1>4 \mathrm{ng} / \mathrm{mL}$ as the threshold value, the specificity of the combined measurement of the three markers can achieve high sensitivity and specificity. The 
diagnostic standard is expressed as TPS $>50 \mathrm{pmol} / \mathrm{L}$, $\mathrm{CEA}>25 \mathrm{pmol} / \mathrm{mL}$ and CYFR21-1 $>120 \mathrm{pmol} / \mathrm{L}$ after concentration unit conversion in order to support the iBioSim simulation.

\subsection{Threshold of TPS, CEA and CYFR21-1}

For the digital circuit, efficient logic decision function relies on optimized selection of the threshold voltage of high and low level and other related circuit parameters, without which the function of the circuit can be inaccurate or interfered by noises. Similarly, the detection system needs research on how to optimize its threshold concentration as well. Appropriate input threshold can ensure accuracy of the results and certain anti-

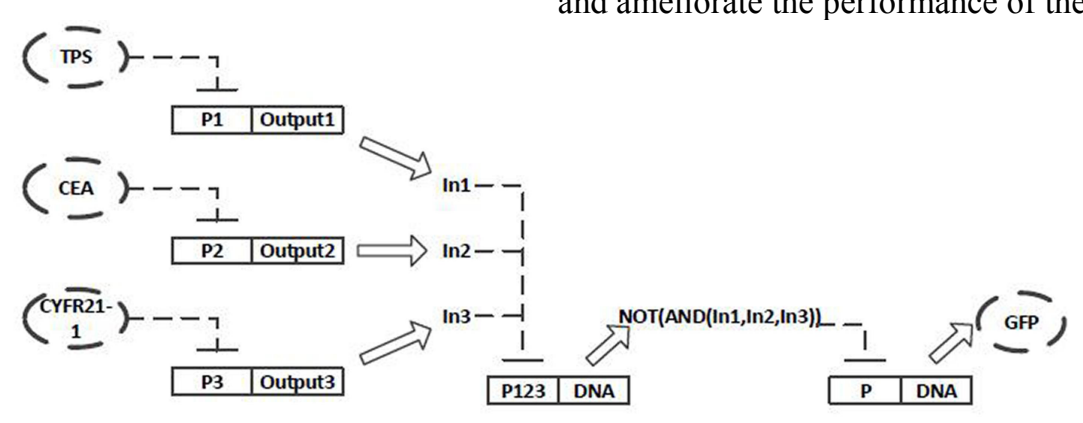

Figure 3. The Whole Design of The Detection Circuit

\subsection{Experimental Result}

First, we intend to prove the discussion about markers' threshold in section 4.1. In this design, we take TPS > $50 \mathrm{pmol} / \mathrm{L}$, CEA $>25 \mathrm{pmol} / \mathrm{mL}$ and CYFR21-1 > $120 \mathrm{pmol} / \mathrm{L}$ as the joint detection standard, which can achieve high sensitivity and specificity of the lung cancer

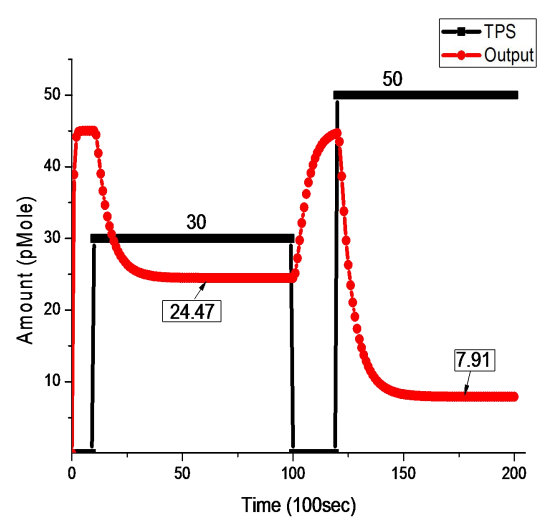

a)The single-index circuit output of TPS interference function. In the circuit we discuss in this paper, parameters of the input module of the single marker detection circuit needs to be adjusted, while the parameters of the output module also need to be adjusted to ensure the normal operation of the circuit and adjust the amplitude adjusting of the circuit. The simulation results are displayed in section 4.2.

Based on the simulation results, we can conclude that after modulation and optimization of the single marker detection circuit, the output amplitude of the three modules is relatively close and the output level to threshold input is also close, avoiding the situation that a single marker detection has too high or low output, which is more conducive to determine the judgment concentration levels, improve the accuracy of the results, and ameliorate the performance of the entire system. detection. So we need to adjust parameters of each singleindex circuit to ensure the output in the same level.

Figure 4 and Table 1 show the output results of this single-index circuit.

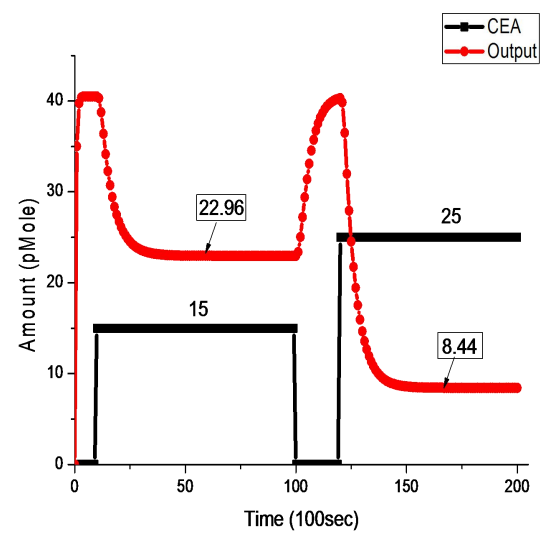

b) The single-index circuit output of CEA 


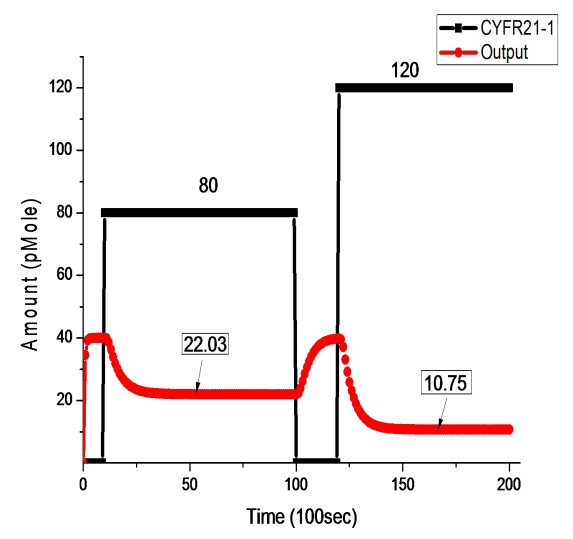

c) The single-index circuit output of CYFR21-1

Figure 4. Results For Threshold Selection of Tps, Cea and Cyfr21-1

Table 1. Results For The Threshold of Tps, Cea and Cyfr21-1

\begin{tabular}{cccc}
\hline Tumor marker & $\begin{array}{c}\text { Amount-of-substance } \\
\text { concentration }\end{array}$ & Output value & Judgment \\
\hline CEA & 15 & 22.96 & High \\
& 25 & 8.44 & Low \\
TPS & 30 & 24.47 & High \\
& 50 & 7.91 & Low \\
CYFR21-1 & 80 & 22.03 & High \\
& 120 & 10.75 & Low \\
\hline
\end{tabular}

Based on the above discussion and analysis, experiment is designed to test the performance of the whole joint system under the condition that TPS > $50 \mathrm{pmol} / \mathrm{L}, \quad \mathrm{CEA}>25 \mathrm{pmol} / \mathrm{mL}$ and CYFR21-1 > $120 \mathrm{pmol} / \mathrm{L}$ are defined as the detection threshold. Figures 5 depict the experimental results of the whole design. Once a marker doesn't reach the threshold value, the output will get close to zero. The output GFP is produced and judged to be high only when all the three input markers TPS, CEA and CYFR21-1 are at high levels.

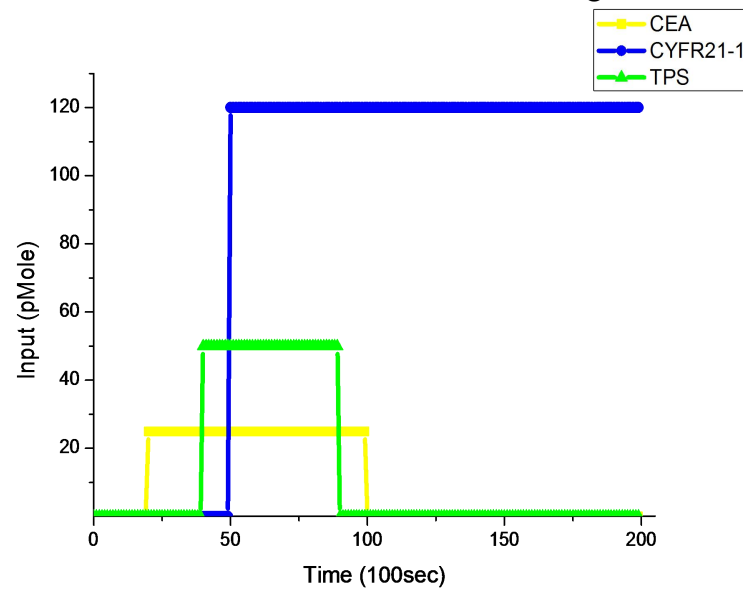

a) input
Simulation results show that the type of circuit structure can achieve detection of the tumor markers of lung cancer with high veracity and real time quality. Compared to the relatively extensive detection method,uch as medical imaging technology, the genetic circuit we proposed can provide precise detection result at each stage of lung cancer. According to the simulation result, it has outstanding advantages in the treatment cost and reliability of the treatment, which can provide the realtime data of the markers related to the lung cancer.

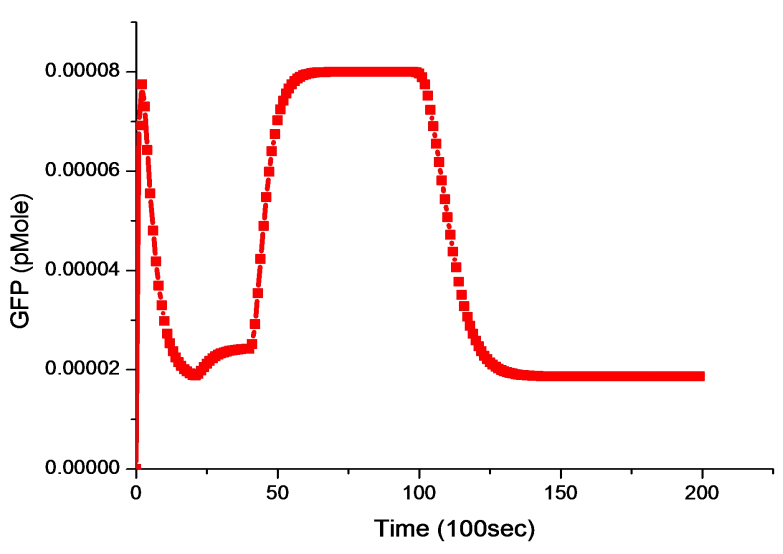

b)output

Figure 5. Simulation Result of The Whole Joint Detection Circuit 


\section{Conclusion}

This paper introduces a novel system of gene circuit to achieve high efficiency and economical early-stage prediction of lung cancer. The design contains two parts: single marker detection circuit and integration detection circuit. Based on the SBML model of genetic gate, the whole system is modelled and simulated by iBioSim. Our design uses the inverter-based single-marker detection circuit to realize concentration detection of each marker, and presents the integration circuit to perform logic calculation of the three outputs from the single-marker circuits to finish the joint detection. The joint system innovatively introduces the analysis on the transmission properties in the single-index circuit and the threshold selection in the integration circuit to increase the whole system's accuracy and reliability. The simulation result shows that the whole system can implement the detection and early warning of lung cancer with good realtime performance and high accuracy. The design can also provide reference for further treatment.

\section{Acknowledgment}

This research is supported by the National Science Foundation of China (Grant No. 81571142), the National Science Key Foundation of China (Grant No. 61232009), the National Science Foundation of China (Grant No. 60973106), Astronautic support Fund (Grant No. 374007), and National 863 Project of China (Grant No. 2011AA010404), guidance and modification of Xia Li.

\section{References}

1. Organization: International Agency for Research on Cancer, GLOBOCAN 2012: Estimated Cancer Incidence, Mortality and Prevalence Worldwide in 2012.

http://globocan.iarc.fr/Pages/fact_sheets_population. aspx

2. Amorín Kajatt E. Lung cancer: a review of current knowledge, diagnostic methods and therapeutic perspectives, Revista Peruana De Medicina Experimental Y Salud Pública, 30, 1: 85-92(2013)

3. Vansteenkiste J, Dooms C, Mascaux C, et al. Screening and early detection of lung cancer. Cancer Journal, 10, 1: 320-327 (2011)
4. Jimeno A, Hidalgo M. Molecular biomarkers: their increasing role in the diagnosis, characterization, and therapy guidance in pancreatic cancer. Molecular Cancer Therapeutics, 5, 4: 787-96 (2006)

5. Grunnet M, Sorensen J B. Carcinoembryonic antigen (CEA) as tumor marker in lung cancer. Lung Cancer, 76, 2: 138-143 (2012)

6. Okamura K, Takayama K, Izumi M, et al. Diagnostic value of CEA and CYFRA 21-1 tumor markers in primary lung cancer. Lung Cancer, 80, 1: 45-49 (2013)

7. De Rubertis G, Davies S W. Genetic circuit design from an electronics perspective. Molecular, Cellular and Tissue Engineering, 2002. Proceedings of the IEEE-EMBS Special Topic Conference on IEEE, 145-146 (2002)

8. Chen C, Sun W, Zheng B, Mao D, Liu W. An incremental approach to closest pair queries in spatial networks using best-first search. In International Conference on Database and Expert Systems Applications, Toulouse, France, 6861:136143(2011)

9. Brophy J A, Voigt C A. Principles of genetic circuit design. Nature Methods, 11, 5: 508-520 (2014)

10. Weiss R, Basu S, Hooshangi S, et al. Genetic circuit building blocks for cellular computation, communications, and signal processing. Natural Computing, 2, 1: 47-84 (2003)

11. Gardner T S, Cantor C R, Collins J J. Construction of a genetic toggle switch in Escherichia coli. Nature, 403, 6767: 339-342 (2000)

12. Anderson J C, Voigt C A, Arkin A P. Environmental signal integration by a modular AND gate. Molecular Systems Biology, 3, 1: 133 (2007)

13. Tamsir A, Tabor J J, Voigt C A. Robust multicellular computing using genetically encoded NOR gates and chemical 'wires'. Nature, 469, 7329: 212-215 (2011)

14. Moon T S, Lou C, Tamsir A, et al. Genetic programs constructed from layered logic gates in single cells. Nature, 491, 7423: 249-253 (2012)

15. Chau A. H, Walter J M, Gerardin J, et al. Designing synthetic regulatory networks capable of selforganizing cell polarization. Cell, 151, 2: 320-332 (2012)

16. Wang X, Yuan GQ, Wang X, Zhao ZX, Wang K. Communication and Monitor of Breast Cancer Signal in the Pulse-Output Genetic Circuit Network. Science China, 57, 3: 1-10 (2014) 